\title{
INFLUENCIAS ARTÍSTICAS E INTERCAMBIO DE ARTISTAS ENTRE NÁPOLES, LAS ISLAS OCCIDENTALES ITÁLICAS Y LAS ISLAS ORIENTALES HISPÁNICAS ${ }^{1}$
}

\author{
Antònia Juan Vicens ${ }^{2}$
}

\begin{abstract}
RESUMEN
El presente artículo pretende analizar las influencias artísticas y el intercambio de artistas entre Nápoles, las islas occidentales itálicas y las islas orientales hispánicas -concretamente de Mallorca-, centrándose en el terreno de la arquitectura y la escultura. Se argumentará a partir del estudio de los maestros y talleres mallorquines que trabajaron en tierras itálicas y de la sucesiva presencia en Mallorca de artesanos procedentes de Italia, en especial de las islas de Sicilia y Cerdeña. Estos últimos se afincarán en la isla balear como aprendices de un maestro local para perfeccionar el oficio y, así, posteriormente, poder penetrar en los circuitos artísticos isleños, incluso formar su propio taller. Las aseveraciones que de ello se desprendan se clarificarán con ejemplos arquitectónicos y escultóricos, aparte de los documentales, que ilustren la penetración e interrelación de elementos artísticos. El marco cronológico se acotará a la segunda mitad del siglo XV i principios del XVI, periodo en que se incentivaron las relaciones entre ambos territorios geográficos debido, entre otras cosas, a las conquistas de Alfonso el Magnánimo.
\end{abstract}

Palabras clave: Escultura gótica, arquitectura gótica, lapiscida, talleres, Sagrera, Mallorca, Italia.

\begin{abstract}
This paper deals with the respective artistic influences and the traffic of artists between Naples, western Italian islands and eastern Hispanic islands - especially Majorca-. We shall centre the study on architecture and sculpture, so analysing Majorcan masters and
\end{abstract}

Fecha de recepción: 1 de octubre de 2009. Fecha de aceptación: 29 de diciembre de 2009

1 Este trabajo se inserta en el proyecto de investigación Guillem Sagrera y la escultura arquitectónica del siglo $X V$, dirigido por la Dra. Sebastiana María Sabater Rebassa y financiado por la Secretaría de Estado de Investigación, referencia HUM2005-04035. Ha sido posible, además, gracias a la concesión de una beca predoctoral por la Conselleria d'Economia, Hisenda i Innovació del Govern de les Illes Balears.

2 Universitat de les Illes Balears. Email: antonia_jv@terra.es. 
workshops who worked in Italia and the subsequent presence in Majorca of Italian artists, mostly those ones from Sicily and Sardinia. They will come to Majorca to remain with a local master to improve their job, so then they could accede at the professional circuits in the island and open their own workshop. Architectonical, sculptural and documentary examples will clarify it; also will illustrate the penetration and interrelation of artistic elements. We shall delimitate the chronological period at second half of $15^{\text {th }}$ century and beginnings of $16^{\text {th }}$ when the links between both geographical regions were closer because of King Alfonso's (Magnanimous) conquests.

Keywords: Gothic sculpture, gothic architecture, lapiscida, workshops, Sagrera, Majorca, Italy.

A lo largo de toda la Edad Media, la Corona de Aragón tuvo mayor contacto con las tierras itálicas que con los reinos hispánicos, lo cual resulta paradójico si se tiene en cuenta que por cercanía geográfica debiera haber sido a la inversa. Ello se explica por el destacado desarrollo comercial-marítimo que experimentó la corona catalano-aragonesa y por la política expansionista que emprendió en el Mediterráneo al verse limitada por el oeste-reino de Castilla-, y por el norte - invasión francesa en los territorios de Occitania - . Así, Pedro el Grande empezó la conquista de territorios italianos invadiendo Sicilia en 1282, Alfonso el Benigno hizo lo mismo con Cerdeña en 1323-27 y Alfonso el Magnánimo completó estas conquistas con su entrada triunfal en Nápoles en 1442 donde asentó su corte.

Fue precisamente en tiempos de Alfonso el Magnánimo cuando se intensificó el intercambio de artistas entre las tierras itálicas y las islas orientales hispánicas, en especial con Mallorca. Por tanto, será entonces cuando se afianzarán las influencias artísticas entre ambos territorios que se prolongarán hasta bien entrado el siglo XVI. Estos hechos han sido lo que ha llevado a fijar la investigación en el periodo cronológico que se extiende desde mediados del siglo XV hasta principios del XVI.

Los objetivos propuestos se centrarán en demostrar cuales fueron las influencias y el intercambio de artistas generados en el terreno de la arquitectura y la escultura. Se argumentará a partir del estudio de los maestros y talleres mallorquines que trabajaron en tierras itálicas y de la sucesiva presencia en Mallorca de artesanos procedentes de Italia, en especial de las islas de Sicilia y Cerdeña. Éstos últimos se afincarán en la isla balear como aprendices de un maestro local para perfeccionar el oficio y así, posteriormente, poder penetrar en los circuitos artísticos isleños e incluso formar su propio taller. Las aseveraciones que de ello se desprendan se clarificarán con ejemplos arquitectónicos y escultóricos, aparte de los documentales, que ilustren la penetración e interrelación de elementos artísticos.

\section{EL TALLER DE GUILLEM SAGRERA COMO DETONANTE DEL PROCESO}

Guillem Sagrera es el arquitecto y escultor más destacado de todo el panorama artístico de la Corona de Aragón durante la primera mitad del siglo XV. La influencia de su 
taller incluso se extiende a lo largo de toda la segunda mitad, sobretodo en el caso de Mallorca, de donde Sagrera era oriundo. ${ }^{3}$

Guillem Sagrera, tras haber intervenido en obras de tal magnitud como la catedral de Palma de Mallorca, la catedral de San Juan de Perpiñán y de haber iniciado la Lonja de Palma, ${ }^{4}$ uno de los proyectos de arquitectura civil más relevantes de las tierras mediterráneas, fue requerido en 1446 por el rey Alfonso el Magnánimo para ponerse al frente de la reforma de su nueva residencia en Nápoles, el Castel Nuovo. Sagrera llegó a la ciudad partenopea en febrero de 1447 y junto a él también se desplazaron miembros de su taller. La actividad del artista mallorquín en tierras napolitanas se extendió hasta el año de su muerte, en 1454, cuando todavía no había concluido la obra por la cual se había trasladado allí. Alfonso el Magnánimo decidió confiar la finalización de ésta a Jaume y Joan Sagrera, hijo y primo del difunto artista, respectivamente, con lo cual se pone de manifiesto la total confianza que había puesto el monarca en el taller del maestro mallorquín.

El prestigio de que gozaba el taller de Sagrera sin duda debió incrementarse tras la expresa llamada del rey. Por ello se encuentran en la Campania, en los territorios que formaron parte en su día del Reino de Nápoles, diversas obras documentadas de su taller y otras que sin estarlo se le pueden afiliar estilísticamente. Pueden señalarse como ejemplos el castillo de Gaeta, obra documentada de Antoni Sagrera, el palacio de Parascandolo en Carinola, el palacio Antignano, en Capua y otros en Nola y Sessa. ${ }^{5}$

En Nápoles también se contabiliza la presencia de otros artistas mallorquines que sin formar parte del taller de Sagrera trabajaron a las órdenes del rey; es el caso de Mateu Forsimanya documentado en $1455 .{ }^{6}$ De él se sabe que intervino en las obras del palacio de Marino Marzano en Carinola y se supone que también lo hizo en el palacio ducal de Sessa Aurunca y en el castillo de los Gaetani, en Fondi. ${ }^{7}$

Otro de los lapiscidae ${ }^{8}$ documentado en Nápoles es Pere Mersa. No se sabe con certeza si era napolitano o mallorquín porque las fuentes no informan sobre el particular, pero lo más probable es esto último puesto que tenía un hermano, Bernat, trabajando en

3 La historiografía ha tratado extensamente la figura del arquitecto y escultor mallorquín. Para una aproximación a su vida y obra véase a modo de ejemplo: H. E. WETHEY; "Guillermo Sagrera” en The Art Bulletin, XXI. A quarterly publ. by the College Art Association of America. 1939, pp. 44-60. G. ALOMAR; Guillem Sagrera y la arquitectura del siglo XV. Blume. Barcelona. 1970. M. R. MANOTE; L'escultura gòtica catalana de la primera meitat del segle XV a la Corona d'Aragó: Pere Joan i Guillem Sagrera. Universitat de Barcelona. Barcelona (Tesis doctoral inédita. Microfilmada). T. SABATER; "Guillem Sagrera, arquitecto y escultor" La Lonja de Palma. Govern de les Illes Balears. Olañeta. Palma. 2003, pp. 57-77.

4 Sobre los problemas que le causó este proyecto y el pleito que de él se derivó con el Colegio de la Mercaderia veáse M. R. MANOTE; "El contrato y el pleito de la Lonja entre Guillem Sagrera y el Colegio de mercaderes de Ciutat de Mallorca" en BARRAL I ALTET, X. (coord.); Artistes, Artisans et production Artistique au Moyen Age. Vol. 1 (Les Hommes). Picard. Paris. 1986, pp. 577-589.

5 G. ALOMAR; Guillem Sagrera... Pág. 206 y A. CIRICI; L'art gòtic català. L'arquitectura als segles XV $i$ XVI. Edicions 62. Barcelona. 1979, p. 60.

6 ARM, EU 10, f. 148.

7 M. ROSSI; L'altro rinascimento. Architettura meridionale del '400. Liguori Editore. Napoli. 2007, p. 156 y 167.

8 Debido a la indefinición terminológica en la documentación medieval sobre arquitectos y escultores, se optará por el uso del término latino lapiscida/ae que engloba a todos aquellos que trabajaban la piedra. 
Mallorca y desempeñando cargos de importancia como el de obrer de la vila, ${ }^{9}$ lo que viene a ser lo mismo que el maestro de las obras de la ciudad. ${ }^{10}$ Además figura en un documento de 1467 firmado en Mallorca donde actúa como testigo junto a Mateu Forsimanya. ${ }^{11}$ Gracias a este documento Pere Mersa puede ser adscrito a la órbita de Forsimanya, de quien como se ha dicho pasó algunas temporadas en Nápoles. Seguramente Pere se debió trasladar allí en un momento indeterminado tal y como lo hicieron el mismo Mateu y otros mallorquines, regresando posteriormente a su ciudad natal. En el caso de Pere el retorno se debió a la petición que su hermano Bernat dirigió en nombre suyo a la Universitat ${ }^{12}$ en 1479, mientras todavía residía en Nápoles junto a su familia, para que se le concediese una franquesa de prevere $^{13}$ que le fue otorgada con la condición de que se trasladase de inmediato a Ciutat de Mallorca junto con sus bienes y su familia. ${ }^{14}$

La presencia de artistas mallorquines no se limita a Nápoles sino que también se registra en Sicilia y Cerdeña. Algunos miembros del taller sagreriano, una vez finalizada su labor en Campania, no regresaron directamente a Mallorca sino que pasaron antes por estas islas. Es el caso de Joan Sagrera, primo de Guillem, que intervino en 1459 en el palacio arzobispal de Palermo. ${ }^{15}$ En torno a los años noventa del siglo XV se tiene también constancia de otro miembro de la familia Sagrera en Palermo, se trata de Vincenzo, cuyo parentesco concreto es todavía hoy desconocido aunque su linaje es prueba evidente de la presencia de la saga en tierras sicilianas. ${ }^{16} \mathrm{Al}$ respecto, Alexandre Cirici le consideró uno de los difusores de la arquitectura catalana en Sicilia. ${ }^{17}$ El también mallorquín Joan Casada residió en Siracusa en torno a 1477 y posteriormente se desplazó a Palermo donde intervino en obras de considerable importancia, como el palacio Abbatelli, coincidiendo en algunas de ellas con artistas italianos de la talla de Mateo Carnilivari. ${ }^{18}$ Igualmente se

9 ARM, EU 19, f. 74v.

10 A. JUAN VICENS; "La figura del 'Maestro de las obras de la ciudad' de una Urbs medieval: el caso de Mallorca” ARIZAGA BOLUMBURU, B., SOLÓRZANO TELECHEA, J. A. (Coord.); Construir la ciudad en la Edad Media. Instituto de Estudios Riojanos (IER). Logroño. 2010. Págs. 357-371.

11 ARM, Prot. M-255, f. 117v.

12 La Universitat era el nombre que recibía el régimen municipal que se estableció según la Carta de Privilegis i Franqueses (1249) en la isla de Mallorca después de la conquista catalano-aragonesa.

13 Las franqueses de prevere consistían en eximir de impuestos durante un tiempo de diez años, del mismo modo que lo estaban los presbíteros, a aquellos a quienes se concedía. Eran un reclamo para extranjeros que deseaban establecerse en la ciudad, del mismo modo que eran un medio utilizado por la Universitat para atraer a gente de fuera según sus necesidades o intereses. Un ejemplo de ello es la concesión que se le hizo en 1467 a Mateu Forsimanya cuando éste residía todavía en Nápoles para que volviese a Mallorca a ponerse al frente de las obras del hospital: ARM, EU 13, f. 100v-102, documento publicado por A. PONS; "Miscelànea de documents" en BSAL, 26. Palma. 1953, pp. 212-213.

14 ARM, EU 16, f. 60-60v. Documento referenciado por O. VAQUER; "Immigrants a Mallorca a la segona meitat del segle XV" en BSAL, 55. 1999, p. 358.

15 Para hacer esta aseveración, Gabriel Alomar se basa en un documento publicado por Filippo Melli en Matteo Carnilivari e la architettura del quattro e del cinquecento in Palermo, donde un tal Joan Cibrera había esculpido la tracería de un ventanal de estilo sagreriano. Alomar supone que Cibrera es una deformación del nombre Sagrera. G. ALOMAR; Guillem Sagrera... p. 212.

16 G. ALOMAR; Guillem Sagrera... p. 226.

17 A. CIRICI; L'art gòtic català. L'arquitectura als segles XV i XVI. Edicions 62. Barcelona. 1979, p. 55.

18 G. ALOMAR; Guillem Sagrera... Pág. 240 y M. R. NOBILE; "La arquitectura en la Sicilia aragonesa (1282-1516)" Una arquitectura gótica mediterránea. Vol. II. Generalitat Valenciana. Conselleria de Cultura i Educació. Valencia. 2003, pp. 24-25. 
tiene constancia de un tal Pietro Mayorchino que fue uno de los fundadores de la corporación de constructores de Palermo en 1487. ${ }^{19}$ Todos estos datos confirman que la presencia mallorquina en Sicilia era habitual y no un caso aislado.

Con respecto a Cerdeña, los ejemplos son más escasos pero aun así puede contarse alguno como el de Pere Vilasclar de quien sabemos por un documento indirecto que se encontraba en la villa de Alguer en 1490. ${ }^{20}$ Igual que los Sagrera, los Vilasclar eran una saga de lapiscidae muy destacada en el panorama artístico insular, por lo tanto su presencia en tierras itálicas es también significativa para comprender la posterior presencia en Mallorca de un número elevado de artistas sardos que firmaron carta de aprendizaje con diversos maestros locales, algunas de ellas con miembros de esta misma familia.

\section{LLEGADA Y ASENTAMIENTO DE ARTISTAS ITALIANOS EN MALLORCA}

Mallorca, debido a su posición estratégica dentro del Mediterráneo y al tráfico mercantil, fue durante toda la Edad Media cruce de caminos y parada casi obligatoria en las rutas comerciales. Por esta razón fundamental fue tierra de acogida de mercaderes, navegantes y artesanos, entre otros colectivos. Los italianos constituyeron uno de los grupos más numerosos por los motivos históricos que se han señalado con anterioridad. Por ello ya encontramos artistas oriundos en la primera mitad del siglo XV. Algunos ejemplos son el de Andreu Sard que consta trabajando en el libro de fábrica de la catedral de 1426$1427^{21}$ o el del siciliano Jorbado Lopilato, procedente de Trapaní, documentado en $1440^{22}$ y relacionado también con el taller de los Sagrera. No obstante, lo cierto es que durante el siglo XIV y la primera mitad del XV los artistas procedentes del norte de Europa y del actual territorio francés predominaron sobre los italianos. ${ }^{23}$ No fue hasta la segunda mitad del XV y principios del XVI cuando éstos últimos pasaron a serlo. Es entonces cuando en la documentación archivística, en particular en la serie de protocolos notariales del Arxiu del Regne de Mallorca, abundan las referencias a artistas italianos que se afincaron en tierras mallorquinas.

Los presentes en mayor número son los sardos, de los cuales se ha podido contabilizar un total de doce, seguidos por los sicilianos, de quienes hay constancia de dos. ${ }^{24}$

19 M.R. NOBILE; "La arquitectura en la Sicilia aragonesa.., p. 24. Sin lugar a dudas el apellido "Mayorchino" debía hacer alusión al lugar de donde era oriundo.

20 ARM, Prot. M-626, f. 125. Documento referenciado por M. BARCELÓ; "Notes sobre els Vilasclar, picapedres" en BSAL, 49. Palma. 1993, p. 138, nota 46.

21 J. SASTRE MOLL; La Seu de Mallorca (1390-1430). La prelatura del bisbe Lluís de Prades i d'Arenós. Lleonard Muntaner. Consell de Mallorca. Palma. 2007, p. 557.

22 G. LLOMPART; "Sagreriana Minora” en BSAL 39. Palma. 1983, pp. 407-434, doc. 11.

23 Por lo que respecta al siglo XIV tenemos los ejemplos de Pierre de Guines, procedente de Artois, la saga de los Campredon y Arnau Boas, oriundos del Rosellón, Jean de Valenciennes y Rich Alamant, provenientes de la zona septentrional de Europa y por lo que respecta a la primera mitad del siglo XV, Pierre Auttor, Blai Mauri o Joan de Saboya.

24 Con respecto a los napolitanos, se ha comentado ya el caso de Pere Mersa quien residiendo en Nápoles se trasladó a trabajar a Mallorca aunque lo más probable es que no fuese oriundo de Italia sino de Mallorca sin descartar del todo la posibilidad de un origen itálico. 
Con respecto a los sicilianos, Bartomeu Asquillaix es, cronológicamente, el primer documentado. La primera noticia hallada sobre él data de 1479 - cuando actúa como testigo en el contrato de aprendizaje que firmó con el maestro mallorquín Guillem Vilasclar otro siciliano, Joan Oliver - ${ }^{25}$ aunque su presencia en Mallorca puede retrotraerse a 1477, año en que también firmó un contrato de aprendizaje con el lapiscida mallorquín Pere Cifre por tiempo de cuatro años. Asquillaix rompió el pacto, no respetando el período de tiempo establecido, por lo cual tuvo que pagar en 1480 una indemnización a Cifre de 17 libras. ${ }^{26}$ Es de destacar que un año antes a este hecho, cuando actuó como testigo en el contrato de su paisano, Asquillaix ya aparece denominado con el apelativo de lapisci$d a$, cuando se supone todavía estaría bajo la tutela de Pere Cifre. Esta denominación no tendría razón de ser si aún estuviera en periodo de aprendizaje. Lo más probable es que cuando se concertó con Pere Cifre ya tuviese algunas nociones del oficio, incluso cabe la posibilidad de que ya se encontrase en posesión de grado de oficial y que el motivo que lo llevó a concertarse con él se debiese al interés por profundizar en el oficio o para especializarse en algún aspecto del mismo. Además, haber pasado un período de tiempo con un maestro mallorquín sin duda le facilitaría la entrada en el circuito profesional isleño, con lo cual posteriormente tendría más posibilidades que otro extranjero de recibir encargos de particulares.

Joan Oliver es otro siciliano que en 1479, a una edad comprendida entre los dieciocho y los veinticinco, se contrató con el maestro Guillem Vilasclar ${ }^{27}$ por tiempo de tres años para aprender el oficio de lapiscida. ${ }^{28}$ El caso de Joan Oliver es paradigmático de un artista extranjero que tras haberse trasladado a Mallorca y haber aprendido durante un periodo de tiempo preestablecido con un maestro mallorquín, decidió establecerse definitivamente en la isla y abrir un taller por cuenta propia. Recibió varios encargos, tanto escultóricos como arquitectónicos, ${ }^{29}$ tomó algunos aprendices a su cargo como el sevillano Juan Marín y, además, su prestigió queda constatado por la relación profesional que tuvo con Melchor Tomás, miembro de una importante familia de la nobleza mallorquina. ${ }^{31}$

Por lo que respecta a los lapiscidae procedentes de Cerdeña contamos con la relación nominal, ${ }^{32}$ aunque la información sobre ellos es en general escasa, por lo común limitada a testificaciones en actas jurídicas. Es el caso de Lleonard Parray, de quien sólo tenemos noticia de su actuación como testigo en enero de 1500 en un acta de los mayordomos

25 G. LLOMPART; “Sagreriana Minora”....doc. 13.

26 G. LLOMPART; "Sagreriana Minora”... doc. 14.

27 Recordemos que la familia de los Vilasclar estuvo estrechamente relacionada con el taller de Guillem Sagrera. Fue precisamente Guillem Vilasclar junto con Miquel Sagrera, sobrino de Guillem, quienes se encargaron de la finalización del edificio de la Lonja cuando Guillem Sagrera partió hacia Nápoles.

28 G. LLOMPART; "Sagreriana Minora”... doc. 13.

29 G. LLOMPART; "Maestros albañiles y escultores en el Medievo mallorquín” en BSAL, 49. Palma. 1993, pp. 249-272, doc. 21.

30 G. LLOMPART; "Maestros albañiles y escultores en el Medievo mallorquín”... doc. 22.

31 ARM, Prot. M-660, f. 10v; ARM, Prot. M-660, f. 39v-40; ARM, Prot. M-660, f. 73v y ARM, Prot. T-876, f. 54 .

32 La relación de los sardos presentes en la isla durante la segunda mitad del XV y principios del XVI es la siguiente: Antoni Jordi, Lleonard Parray, Lleonard Arraix (?), Miquel Carbonell, Miquel Amorós, Gabriel Pujol, Domingo Sard, Antoni Orraxi, Ángel de Sácer, Amingo Sucha, Salvador Guiso y Joan Villa Esglesies. 
del oficio de tejedores, ${ }^{33}$ de Lleonard Arraix (?), que lo hace en un débito en $1501,{ }^{34}$ y de Domingo Sard ${ }^{35}$ quien actúa como testigo en un contrato en noviembre de $1505 .{ }^{36} \mathrm{De}$ Antoni Jordi sabemos poco, no obstante cabe destacar un documento de 1502 que lo relaciona con el lapiscida mallorquín Jeroni Ferrer, donde éste actúa como testigo en la firma de un documento de aquél. ${ }^{37}$ Jeroni Ferrer fue un maestro destacado durante la época que nos ocupa y el hecho que actuase como testigo de Antoni indica que al menos se conocían, la relación entre ellos es por ahora desconocida pero no sería de extrañar que hubiesen mantenido estrecho contacto y que incluso hubiesen colaborado en alguna ocasión.

Otros ejemplos de sardos son Gabriel Pujol, procedente de la villa de Alguer, documentado en Mallorca en 1505,38 y Antoni Orraxi, procedente de Cáller, a quien se le concedió un permiso en 1514 para casarse en Mallorca. ${ }^{39}$

Las referencias que tenemos de estos lapiscidae, a pesar de no ser muy significativas a simple vista, informan del elevado número de artistas procedentes de aquella zona geográfica que se afincaban en la isla, suponemos por motivos laborales.

De quienes sí es posible extraer mayor información es de un grupo de cinco sardos que firmaron contratos de aprendizaje y de trabajo con maestros mallorquines. Ángel de Sácer ${ }^{40}$ es uno de ellos: en 1479 se concertó con Pere Llull por tiempo de cuatro años para aprender su oficio. ${ }^{41}$ Otro ejemplo es Amingo Sucha, oriundo de la villa de Asglesies, quien en junio de 1502, cuando contaba con una edad comprendida entre los veintidós y los veinticinco, firmó un contrato de aprendizaje por tiempo de tres años con Joan Sagrera. ${ }^{42}$ Éste, primo de Guillem, estuvo trabajando en Nápoles y Sicilia, y en aquellos años ostentaba el cargo de maestro mayor de la catedral, por lo tanto se trataba de uno de los lapiscidae más reputados de la isla en su época.

Miquel Amorós, de Alguer, se contrato con el yesero Pere Sanxo, en este caso no para aprender el oficio, puesto que él mismo se designa ya como lapiscida, sino para trabajar para él durante medio año, es decir, desde julio de 1500, mes en que se fïrmó el documento, hasta diciembre. ${ }^{43}$ No se especifica en qué consistía el trabajo a realizar pero atendiendo al espacio de tiempo contratado es probable que no se tratase de una labor de gran envergadura. Aproximadamente un año después, en febrero de 1501, se

33 ARM, Prot. T-837, f. 56v.

34 ARM, Prot. P-397, f. 39v-40.

35 En el libro de fábrica de la catedral de Palma de 1427-1428 aparece trabajando un Domingo Sard pero no parece tratarse de la misma persona puesto que la franja cronológica es demasiado extensa. J. SASTRE MOLL; La Seu de Mallorca (1390-1430)..., p. 576.

36 ARM, Prot. T-455, f. 119-122.

37 ARM, Prot. R-772, f. 185-185v. Documento referenciado por O. VAQUER; "Immigrants a Mallorca (15001550)" en BSAL, 54. 1998, p. 122.

38 O. VAQUER; "Inmigrants a Mallorca a la segona meitat del S. XV" en BSAL, 51. 1995, p. 136. Desgraciadamente el autor no ofrece la referencia archivística que, sin duda, nos esclarecería algún aspecto más sobre su persona.

39 O. VAQUER; “Immigrants a Mallorca (1500-1550)"..., p. 129.

40 Supuestamente Sacer hará referencia a Sasari, una de las cuatro provincias en que está dividida la isla en la actualidad.

41 ARM, Prot. P-374, f. 62.

42 ARM Prot. P-316, f. 60.

43 ARM, Prot. P-396, f. 20v. 
ha localizado una noticia que lo relaciona con Joan Sagrera: se trata de un documento mediante el cual Miquel Amorós contrata a un joven llamando Domingo Fàbregues con Joan para que aprenda el oficio de lapiscida. ${ }^{44}$ El vínculo entre ellos no se precisa pero lo que sí resulta de nuevo interesante es constatar la conexión con un miembro de la familia Sagrera.

Joan Sagrera vuelve a aparecer en otra carta de aprendizaje con un sardo, en este caso se trata de Salvador Guiso, de la villa de San Pantaleone, que se contrata con él en marzo de 1503 por espacio de tres años. ${ }^{45}$ Uno de los testigos de este documento es Joan de Villa de Esglesies, es de suponer que se trate de otro sardo procedente de la villa de Iglesias, también relacionado con Joan Sagrera, aunque fuese de forma indirecta.

Ya para finalizar, citar el caso de Miquel Carbonell, procedente de Cerdeña, de quien sabemos a través de un único documento fechado en septiembre de 1504, que se inició en el oficio de lapiscida con el mallorquín Joan Antensa (se desconoce el período de tiempo) y que, con el beneplácito de éste, decidió pasar dos años más junto al también lapiscida Antoni Armengol para aprender el oficio, ${ }^{46}$ es de suponer que para profundizar y perfeccionar el mismo.

De lo expuesto anteriormente con respecto a sardos y sicilianos se han constatado dos aspectos: en primer lugar la elevada cifra de artistas oriundos de las islas occidentales itálicas residentes en Mallorca durante la segunda mitad del siglo XV, especialmente a finales de este siglo y principios del siguiente. En segundo lugar, el considerable número de contratos de aprendizaje y trabajo firmados entre los extranjeros y diversos maestros mallorquines, de los cuales el más significativo es Joan Sagrera, documentado en Nápoles y de quien se presume una estancia en Sicilia.

De todo ello pueden deducirse, básicamente, dos extremos:

El primero, el constante tráfico e intercambio de artistas entre las islas occidentales itálicas y las orientales hispánicas. Son del todo conocidos los continuos contactos y las relaciones comerciales entre Mallorca y Cerdeña, desde donde se importaban, igual que desde Sicilia, abundantes cantidades de trigo para paliar la carestía que de éste siempre padecía Mallorca. ${ }^{47}$ Ello no sólo facilitaba el tráfico y la llegada de mercancías, sino también de personas.

El segundo es el considerable interés que muestran los extranjeros por concertarse con un artista mallorquín mediante contratos de aprendizaje y de trabajo. Este interés puede explicarse por el prestigio que habrían alcanzado los lapiscidae mallorquines en su periplo por tierras itálicas. Resulta significativo que los sicilianos Bartomeu Asquillaix y Joan Oliver aparezcan en Mallorca pocos años después de tener documentada la presencia en Sicília de lapiscidae mallorquines, algunos de los cuales pertenecían al círculo de Sagrera.

44 ARM, Prot. M-590, f. 5v-6.

45 G. LLOMPART; "Sagreriana Minora"... doc. 15.

46 ARM, Prot. S-882, f. 128v.

47 Véase: A. SANTAMARÍA; "El mercado triguero en Mallorca en la época de Fernando el Católico" en VI Congreso de historia de la Corona de Aragón. Madrid. 1959, pp. 379-393 y M. BARCELÓ CRESPÍ; "Relaciones comerciales entre Mallorca y Cerdeña (segunda mitad del siglo XV)" en XIV Crongresso di Storia della Corona d'Aragona. Sassari-Alghero, 19-24 Maggio 1990. Vol. 3. Carlo Delfine editore. Sassari. 1996, pp. 101-121. 
También lo es que la mayoría de sardos se encuentren presentes tras haber constatado a Pere Vilasclar como lapiscide ville del Alguer.

Los maestros mallorquines durante su estancia en tierras itálicas debieron establecer relaciones con artistas oriundos del lugar. Esto y el prestigio que habían obtenido fue el detonante del futuro traslado de los italianos a la isla de Mallorca para aprender y perfeccionar el oficio con maestros autóctonos. Un hecho que avalaría esta hipótesis es la estructura de los contratos de aprendizaje que difiere en dos puntos de los tradicionales:

1) Son los propios aprendices quienes formalizan el documento. La no presencia del padre o tutor que solía ser quien firmaba en nombre del aprendiz, es un indicativo de que éstos era ya personas adultas que decidían contraer libremente los compromisos pactados.

2) La mayoría se contrata por un tiempo inferior al que estipulaban las ordenanzas gremiales y a aquel por el cual lo hacían los menores de edad, con el añadido que algunos ya se definen como lapiscidae, cosa imposible para un aprendiz. Ello significa que ya debían estar en posesión de ciertas nociones del oficio y que lo que pretendían con la firma del contrato era profundizar en él y perfeccionarlo bajo la tutela de un maestro mallorquín de reconocido prestigio.

\section{LAS OBRAS DE ARTE COMO REFLEJO DEL PROCESO}

Las influencias artísticas entre las islas orientales hispánicas (y también Cataluña y Valencia) y las tierras itálicas se perciben no sólo en el tráfico e intercambio de artistas sino también en las obras arquitectónicas y escultóricas de ambos territorios.

Por lo que respecta a las influencias catalanas en la arquitectura italiana, éstas se perciben en las residencias nobiliarias, especialmente en los patios, las loggias y los ventanales, sobre todo en cuanto a elementos decorativos se refiere, como el diseño de tracerías, tanto en arcos como en las ménsulas de apeo.

De los patios destaca la escalera exterior a la catalana que comunica el patio con la planta noble, como se ve en el de los palacios Abbatelli de Palermo, Orologio y Bellomo, en Siracusa.

Las tracerías de los ventanales han sido uno de los elementos que ha llevado a determinados investigadores como Gabriel Alomar a identificar algunas obras italianas como de factura catalano-mallorquina. Todas ellas se caracterizan por un tipo de decoración flamígera delimitada por el intradós de arcos y ventanas que se localiza en la parte superior de los vanos, como puede verse en los ventanales del palacio arzobispal de Palermo o en los del castillo de Gaeta en Fondi. ${ }^{48}$ Si se comparan con los de la Lonja de Palma las semejanzas son evidentes, como también lo es el parecido entre las tracerías que decoraban el antepecho - destruido en 1919 a causa de un incendio pero que conocemos gracias a fotografías antiguas - de la tribuna alta del Castelnuovo, destinada a los músicos, con el de la tribuna del órgano de la capilla de Santa Aïna de la Almudaina. ${ }^{49}$

48 A. GABRIEL; Guillem Sagrera..., pp. 210 y $244-245$.

49 Ibidem, p. 207. 
Por lo que a arquitectura se refiere, una de las tesis principales que defiende Massimo Rossi, a pesar de reconocer la huella que dejaron los artistas catalanes sobre muchas obras arquitectónicas y escultóricas de las tierras itálicas, es que, a diferencia de lo que afirma la historiografía tradicional, la influencia catalana sobre la arquitectura de "Terra di Lavoro" no fue tan importante como se ha sostenido. Según él, las semejanzas estilísticas entre ambas y los elementos decorativos que la constituyen se deben no tanto a un influjo directo como a la manifestación de los elementos que en común tienen todos los edificios que pertenecen al gótico internacional. ${ }^{50}$ Tal vez la tesis de Massimo Rossi sea acertada aunque, igual que gran parte de los historiadores, consideramos que no pueden pasarse por alto los siglos de dominio catalán ni la impronta ejercida sobre la sociedad, el arte y la vida de los italianos. De hecho, la introducción de la arquitectura gótica en lugares como la isla de Cerdeña se debió a ellos. Por tanto, no debe minimizarse el influjo catalanomallorquín en el desarrollo artístico del sur y de las islas occidentales itálicas.

Las influencias no fueron sólo unidireccionales, el intercambio fue mutuo. Por lo que respecta a las islas orientales hispánicas, sobre todo en cuanto al caso de Mallorca, algunas de las obras conservadas en iglesias y museos, en especial esculturas de busto redondo de temática mariana, son reflejo de la incidencia de la plástica italiana sobre la mallorquina. Se trata de obras importadas, las cuales sin duda constituyeron un detonante para la asimilación de postulados artísticos italianos en el ámbito mallorquín. Al respecto puede señalarse un conjunto de cinco vírgenes de mármol datadas entre finales del XV y principios del $\mathrm{XVI}^{51}$ de procedencia suditaliana (bien napolitana o siciliana) que mucho tienen que ver con el taller de los Gaggini.

Se sabe que los Gaggini trabajaron en la escultura del arco del Castel Nuovo y que tuvieron un taller en Sicilia. Es muy probable que conociesen a maestros mallorquines y que mantuvieran relación con ellos durante su estancia tanto en Nápoles como en la isla. Las esculturas que se atribuyen a su taller y que se encuentran actualmente en Mallorca son una prueba más de los contactos e intercambios artísticos entre las dos regiones que se incentivaron tras la estancia de maestros mallorquines en tierras itálicas.

\section{A MODO DE CONCLUSIÓN}

Las relaciones políticas y comerciales entre las regiones itálica e hispánica objeto de estudio propiciaron el continuo intercambio de influencias artísticas durante toda la Edad Media, pero el flujo constante de artistas entre ambas zonas se intensificó tras el traslado a aquellas tierras de Guillem Sagrera, miembros de su taller y otros artistas de origen mallorquín. El prestigio que éstos alcanzaron allí fue el detonante para que un considerable número de lapiscidae extranjeros se trasladase a Mallorca para aprender y perfeccionar el oficio bajo la tutela de un reconocido maestro local.

50 M. ROSSI; L'altro rinascimento. Architettura meridionale del ‘400. Liguori Editore. Napoli. 2007.

51 Dos de ellas, que procedían de la desamortizada iglesia de los carmelitas de Palma, se conservan actualmente en la iglesia del Hospital General; otras se encuentran en la iglesia parroquial de Buñola, en el santuario de Montisión de Porreras y en el Museo Diocesano. 
La documentación archivística que corrobora este hecho se ve complementada por el conjunto de obras arquitectónicas y escultóricas presentes en ambos territorios, las cuales ejemplifican la asimilación e intercambio de postulados artísticos entre el sur de Italia, Sicilia, Cerdeña y la mayor de las islas del archipiélago balear.

\section{SIGLAS}

- ARM: Archivo del Reino de Mallorca.

- BSAL: Boletín de la Sociedad Arqueológica Luliana.

- EU: Extraordinarios de Universidad.

- Prot.: Protocolo notarial. 